\title{
Mitarbeiter des ersten Bandes
}

Univ.-Prof. Dr. W. Aly, Freiburg i. B. Univ.-Prof. Dr. W. Anderson, Dor pat.

Dr. E. Bargheer, Referent imMinisterium für Wissenschaft, Kunst und Volksbildung, Berlin.

Univ.-Prof. Dr. W. Berendsohn, Hamburg.

Dr. H. Diewerge, Riga.

Univ.-Prof. Dr. E. Fehrle, Dezernent im Kultusministerium, Karlsruhe.

Dr. E. Frenkel, Wien.

Dr. F. M. Goebei, Oppein.

Univ.-Prof. Geheimrat Dr. W. Golther, Rostock.

Dr. P. Groth, Schneidemüh1.

Dr. Grunwald, Wien.

Dr. K. Heckscher, Seelze b. Hannover.

Dr. W. Heiligendorff, Stralsund.

Prof. Dr. B. Heller, Buda pest.

Prof. Dr. K. Herold, Karlsbad.

Dr. M. Ittenbach, Frankfurt a. M.

Univ.-Prof. Dr. Jungbauer, Prag.

Dr. G. Kahlo, Uslar.

Priv.-Doz. Dr. K. Kaiser, Greifswald.
Prof. Dr. Künzig, Lahr i. B.

Priv.-Doz. Dr. S. Liljeblad, Lund.

Oberstudiendirektor Dr. Ludwig Mackensen, Berlin-Charlottenburg. Univ.-Prof. Dr. Lutz Mackensen, Riga. Dr. E. Müller, Marktredwitz.

Dr. O. Nossag, Kottbus.

Univ.-Prof. Dr. Petzsch, Ha mburg.

Priv.-Doz. Dr. Peuckert, Breslau.

Studienrat Dr. Pieper, Berlin.

Dr. Schmitz, Breslau.

Referendar Maria Schuir, Sterkrade.

Univ.-Prof. Dr. Schulte-Kemminghausen, Münster.

Univ.-Prof. Dr. S. Singer, Bern.

Dr. K. Spieß, Wien.

Dozent Dr. W. v. Sydow, Lund.

Univ.-Prof. Dr. A. Taylor, Chicago.

Dr. Tegethoff, München.

Univ.-Prof. Dr. Voretzsch, Halle.

Univ.-Prof. Dr. J. de Vries, Leiden.

Dr. Weydt, Frankfurt a. M.

Dr. H. Wetter, Wiesbaden.

Univ.-Prof. Dr. A. Wrede, Köln. 



\section{Friedrich Panzer \\ dem bahnbrechenden Märchenforscher}


